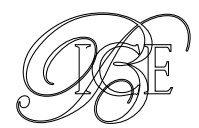

Antonio Moreno-Torres Gálvez*

\section{APLICACIÓN REGULATORIA DEL WACC}

En entornos regulados en los que la competencia dependa del acceso a infraestructuras costosas - en cuanto a su inversión, operación y mantenimiento-, su desarrollo adecuado dependerá de la medida en que sean capaces de atraer la financiación requerida garantizando una rentabilidad «razonable». Como también lo habrá de ser la remuneración que reciba un incumbente como contraprestación por la utilización de sus activos heredados (legacy) por nuevos entrantes. En este artículo se describe breve e intuitivamente la práctica regulatoria de generalizada aceptación que, basada en la metodología del coste medio ponderado de capital (CMPC o WACC en su acrónimo inglés), puede encontrarse en sectores como el de la energía, las telecomunicaciones, el postal o el transporte.

Palabras clave: coste medio ponderado de capital, CMPC, WACC, rentabilidad intrínseca, incentivos, financiabilidad, rentabilidad razonable, regulación, energía, telecomunicaciones.

Clasificación JEL: G32, G38, L51, L90.

\section{Introducción}

El coste medio ponderado de capital es un concepto financiero básico consolidado dentro de la caja de herramientas para la toma de decisiones corporativas de inversión. Como tal, tiene un importante valor regulatorio, y prueba de ello es su uso intensivo por parte de agencias reguladoras sectoriales, como las que en su día se integraron en nuestra Comisión Nacional de los Mercados y la Competencia (CNMC), que la viene aplicando a cuestiones como el cálculo del coste de capital de operadores de telecomunicaciones declarados con

\footnotetext{
* Ingeniero Industrial del Estado y Profesor Asociado en la Universidad Politécnica de Madrid.

Versión de junio de 2021.

DOI: https:/doi.org/10.32796/bice.2021.3137.7255
}

poder significativo de mercado (Resolución de 6 de mayo de 2021) o el cálculo de la tasa de retribución financiera de las actividades de transporte y distribución de energía eléctrica, y regasificación, transporte y distribución de gas natural (Circular 2/2019), por referenciar tan solo dos metodologías fundamentadas en el WACC (Weighted Average Cost of Capital) recientemente actualizadas.

El presente artículo tiene por objeto exponer la metodología del coste medio ponderado de capital aplicada comúnmente en la regulación económica de determinados sectores y actividades. Para ello, comienza planteando un modelo financiero para el cálculo de la rentabilidad intrínseca de un proyecto de inversión para un caso de referencia en el que se recuperen por bloques la totalidad de los costes. Se trata a $\triangleright$ 
continuación el efecto de la estructura financiera y la cuestión de la financiabilidad, introduciéndose el concepto de coste medio ponderado de capital. De la consideración explícita del riesgo a la hora de establecer una rentabilidad razonable surge el método de los comparadores para el cálculo de un WACC regulatorio o nocional, lo que admite numerosos matices metodológicos que se discuten en los subepígrafes centrales de este artículo. Como colofón, se puntualiza cómo las desviaciones con respecto al modelo de referencia de recuperación de costes y con respecto a las condiciones nocionales de financiación se manifiestan en una rentabilidad efectiva distinta de la prospectivamente establecida, con el impacto económico que ello supone. Se finaliza el artículo con un apartado de conclusiones a modo de síntesis.

\section{Modelo cost-plus de referencia}

Supóngase un proyecto de inversión con un coste de inversión (CAPEX) inicial de I y unos costes de operación y mantenimiento (OPEX) anuales OPEX $X_{t}$ a desembolsar al final de cada año de su vida útil, establecida en $n$ años desde su puesta en servicio. Si anualmente se recupera, ya sea vía precios o vía retribución regulada, y por bloques de costes (building blocks), una componente de amortización $A M O R T_{t}^{s}$, unos costes operativos OPEX ${ }_{t}^{s}$ y un margen calculado aplicando una tasa de retribución TRF sobre el valor neto de la inversión al comienzo del año $V N I_{t}^{s}$, se tiene que, para una tasa de descuento $r$, el valor actual neto (VAN) del proyecto será:

$$
\begin{gathered}
V A N=\left[-I-\sum_{t=1}^{n} \frac{O P E X_{t}}{(1+r)^{t}}\right]+\sum_{t=1}^{n} \frac{1}{(1+r)^{t}} . \\
\cdot\left\{A M O R T_{t}^{s}+V N I_{t}^{s} \cdot T R F+O P E X_{t}^{s}\right\}
\end{gathered}
$$

Con un patrón lineal de amortización constante (método alemán) $A M O R T_{t}^{s}=l^{s} / n$ y $V N I_{t}^{s}=I^{s}-(t-1) \cdot I^{s} / n \mathrm{y}$, utilizando la notación habitual $a_{n, r}$ para el valor actual de la renta temporal pospagable unitaria (o anualidad), se puede reescribir:

$$
\begin{gathered}
\text { VAN }=-I-\sum_{t=1}^{n} \frac{\text { OPEX }_{t}-\text { OPEX }_{t}^{s}}{(1+r)^{t}}+\frac{I^{s}}{n} . \\
\cdot\left\{\left(1-\frac{1}{r} \cdot T R F\right) \cdot\left(a_{n, r}-n\right)+n\right\}
\end{gathered}
$$

Si $I=I^{s}$ y OPEX $X_{t}=$ OPEX $_{t}^{s}$ (recuperación total de costes), y teniendo en cuenta que por definición de tasa interna de rentabilidad $(T I R)$ es $\operatorname{VAN}(r=T I R)=0$, se tiene que:

$$
\left(1-\frac{T R F}{T I R}\right) \cdot\left(a_{n, T I R}-n\right)=0
$$

Siendo que $a_{n, T / R} \neq n$, entonces ha de ser $1-T R F / T I R=0$, por lo que, finalmente, resulta que:

$$
T I R=T R F
$$

Es decir, cuando a un proyecto de inversión que recupera la totalidad de sus costes de inversión y operación y mantenimiento se le remunera anualmente con un margen resultante de aplicar a su valor neto de amortización acumulada una tasa de retribución, si se considera un método de amortización lineal sobre valores históricos, la tasa interna de rentabilidad de dicho proyecto coincidirá precisamente con dicha tasa de retribución, sin que dependa de ningún otro parámetro del modelo. Indicar a este respecto que una mayor vida útil $n$, aunque no afecte a la rentabilidad, sí lo hace sobre la liquidez en el corto plazo debido a la laminación de ingresos que provoca.

Una consecuencia directa de que la tasa de retribución se manifieste tal cual como de rentabilidad es la existencia de un incentivo a la $D$ 
sobreinversión por encima del nivel óptimo (efecto Averch-Johnson), que puede mitigarse estableciendo techos máximos a la inversión.

Antes de pasar a discutir en los siguientes epígrafes cuál ha de ser el valor adecuado para la tasa de retribución TRF, cabe preguntarse cómo, en ausencia del acicate de la competencia, podría mejorarse la rentabilidad intrínseca del proyecto. En un modelo regulatorio del tipo Rate-of-Return (RoR) sobre una base regulatoria de activos (Regulatory Asset Base, -RAB-) y retribución individualizada que incorpore incentivos a la eficiencia, los valores $l^{s}$ y OPEX ${ }_{t}^{s}$ estándares 0 de referencia -típicamente expresados en términos de valores unitarios- se corresponderán con los de una empresa eficientemente gestionada, de manera que su mejora resultará en una fuente de rentabilidad extra para el proyecto. Otros incentivos habituales en modelos cost-plus son los de extensión de la vida útil (EVU) -recuperación de OPEX una vez vencida la vida útil del proyecto y en tanto que batan al estándar-, los de mejora de la calidad del servicio o los asociados a mejora de la productividad — por la disminución interanual de los OPEX, en casos de reconocimiento de costes auditados-. Estos últimos, en la regulación por techos de precios o ingresos en un periodo (price-cap o revenue-cap), revisten la forma de la clásica regla $I P C-X$ de indiciación.

\section{Efecto de la estructura financiera}

En el apartado anterior se ha calculado la rentabilidad intrínseca de un proyecto de inversión que recupera sus costes omitiendo cualquier consideración relativa a su financiación. Cuestión diferente es el aseguramiento de dicha financiación (financiabilidad), que pasa por que el proyecto de inversión genere un margen que retribuya adecuadamente a sus financiadores, ya sean ajenos o propios.

Desde el punto de vista práctico, la separabilidad de las decisiones de inversión y financiación se traduce en que la valoración financiera de un proyecto de inversión puede hacerse descontado sus flujos de caja libres, esto es, estimados suponiendo que el proyecto se financia en su totalidad con fondos propios, si se utiliza como tasa de descuento el coste medio ponderado de capital (CMPC o WACC, por sus siglas en inglés) nominal después de impuestos definido como:

$$
W A C C=\frac{D}{D+F P} \cdot r_{D} \cdot(1-T)+\frac{F P}{D+F P} \cdot r_{F P}
$$

en donde $D$ es la deuda, $F P$ los fondos propios, $r_{D}$ y $r_{F P}$ sus respectivos costes y $T$ el tipo impositivo.

De acuerdo con la metodología VAN, y como consecuencia de los atributos de la tasa de descuento así definida (nominal y después de impuestos), los flujos de caja libres habrán de ser también, además de esperados e incrementales, después de impuestos y nominales, esto es, expresados en unidades monetarias corrientes sin que se ajusten por la inflación esperada puesto que, de expresarse aquellos en unidades monetarias constantes (valores reales vs nominales), sería por coherencia necesario utilizar una tasa de descuento expresada también en términos reales.

En una explicación intuitiva, con una inversión I en un proyecto que se espera que genere a perpetuidad los mismos ingresos -flujos de caja operativos netos- anuales manteniendo su nivel de endeudamiento y el mismo mix de financiación entre deuda $D$ y fondos propios $F P$ que la empresa que lo acomete, se tendrá que las financiaciones ajena y propia requeridas $D$ 
ascenderán a $[D /(D+F P)] \cdot /$ y $[F P /(D+F P)] \cdot l$, respectivamente. Si el proyecto vale la pena, los ingresos que genere habrán de cubrir al menos el coste explícito de los intereses devengados después de impuestos $[D /(D+F P)] \cdot l \cdot r_{D} \cdot(1-T)$ y el coste implícito o de oportunidad de la rentabilidad esperada por los accionistas $[F P /(D+F P)] \cdot l \cdot r_{F P}$. Es decir, habrá de ser:

$$
\begin{aligned}
& \text { Ingresos }>\left[\frac{D}{D+F P} \cdot l\right] \cdot r_{D} \cdot \\
& \cdot(1-T)+\left[\frac{F P}{D+F P} \cdot l\right] \cdot r_{F P}
\end{aligned}
$$

de modo que, dividiendo ambos miembros por $I$, y teniendo en cuenta que la rentabilidad del proyecto viene dada por el cociente entre los ingresos que genera y la inversión $(r=$ Ingresos $/ l)$, se tendrá que:

$$
r_{\text {min }}=\frac{D}{D+F P} \cdot r_{D} \cdot(1-T)+\frac{F P}{D+F P} \cdot r_{F P}
$$

es la rentabilidad mínima que habrá de proporcionar el proyecto para ser financiado (condición de financiabilidad). A este valor se denomina CMPC, puesto que formalmente es una media ponderada de los costes de la deuda después de impuestos $r_{D} \cdot(1-T)$ y de los fondos propios $r_{F P}$ utilizando como pesos respectivos los tamaños relativos de la deuda $D /(D+F P)$ (ratio de apalancamiento) y de los fondos propios $F P /(D+F P)$.

Puede ya dar una interpretación económica clara al esquema retributivo por bloques de costes contemplado en el epígrafe anterior. Siendo la condición de aceptación de un proyecto que su tasa interna de rentabilidad TIR supere al coste de oportunidad, y siendo el $W A C C$ dicho coste de oportunidad, en el umbral $(V A N=0)$ sería $T I R=$ WACC y, por tanto, en el modelo base de recuperación total de costes de capital CAPEX y operación y mantenimiento OPEX en el que TIR $=T R F$ habrá de ser, antes de impuestos:

$$
T R F=W A C C_{A I}=\frac{W A C C}{1-T}
$$

o, con su acrónimo español, TRF = CMPCAI. La tasa de retribución tendrá, pues, motivación financiera, y el margen aplicado, naturaleza de coste del capital.

Con carácter general, para el cálculo regulatorio del coste de capital anual correspondiente a cada ejercicio se aplicará el $W A C C_{A I}$ como tasa de retribución financiera sobre el valor neto de la inversión - valor bruto menos amortización acumulada-, de forma que $C C_{t}^{S}=V N I_{t}^{S} \cdot T R F=V N I_{t}^{S} \cdot W A C C_{A l}$. Ha de tenerse en cuenta entonces que en el modelo cost-plus habrán de omitirse los gastos financieros, para evitar así su doble reconocimiento al formar estos ya parte del WACC.

En las condiciones anteriores, que la $T I R$ del proyecto coincida con su WACC dependerá del patrón de amortización -lineal o no-, del criterio de valoración de activos - costes contables históricos o valores corrientes-y de la existencia o no de incentivos o penalizaciones. En este sentido, otro patrón transparente (en tanto que TIR $=T R F$ ) utilizado en la práctica es el de amortización acelerada con coste de inversión anualizado constante (método francés), en el que:

$$
\text { CAPEXS }=\left.\frac{\text { WACC }}{1-\frac{1}{(1+W A C C)^{n}}} \cdot\right|^{S}
$$

Este método francés de anualidad constante no es sino una particularidad del más genérico de amortización variable o anualidad con tendencia (tilted annuity), en el que CAPEX $_{t+1}^{s}=$ CAPEXs $\cdot(1+\alpha) \mathrm{y}$, por tanto: 


$$
C A P E X_{t}^{s}=\frac{W A C C-\alpha}{1-\left(\frac{1+\alpha}{1+W A C C}\right)^{n}} \cdot V R_{t-1}^{s}
$$

con:

$$
V R_{t-1}^{s}=l^{s} \cdot(1+\alpha)^{t-1}
$$

observándose cómo, en efecto, cuando $\alpha=0$, se reproduce el caso particular del método francés.

En la expresión anterior, $V R_{t-1}^{s}$ representa el valor de reposición de la inversión inicial $s^{s}=V R_{0}^{s}$ al comienzo del periodo $t$, en una hipótesis de variación constante $\alpha$ (tilt, expresada en tanto por uno) del coste del activo. Se emula así una contabilidad a costes corrientes. Que la evolución temporal de CAPEXs sea la misma que la del valor de reposición de la inversión permitirá a un incumbente seguir siendo competitivo frente a futuros nuevos entrantes. En general, la tendencia en precios podrá ser creciente $(\alpha>0)$ o decreciente $(\alpha<0)$.

Con respecto a la notación utilizada hasta ahora, precisar que algunos lectores preferirán, por serles familiar, la que usa $R I_{t}$ («retribución a la inversión», que incluye componentes de amortización y de coste de capital) y $R O M_{t}$ («retribución por operación y mantenimiento») en lugar de CAPEX $X_{t}$ y OPEX $X_{t}^{s}$, respectivamente.

\section{Método de los comparadores}

En la valoración financiera de proyectos de inversión mediante descuento de flujos de caja esperados, el riesgo se incorpora en la tasa de descuento. Mientras no se disponga de una cuantificación particular de esta para el proyecto en cuestión (por ser acometido por una empresa no cotizada o, aun siendo cotizada, por ser de un riesgo diferente del promedio de la empresa que lo acomete), resulta adecuado un método de comparación (enfoque de mercado vs individual) que construya una referencia nocional.

Desde el punto de vista de los financiadores, son los propietarios - y no los prestamistas- los que en general soportan el riesgo y a los que, por tanto, se habrá de compensar adecuadamente por este concepto. Así, la componente de rentabilidad exigida a los fondos propios $r_{F P}$ del WACC será, en tanto que coste de oportunidad, el rendimiento esperado de un activo con el mismo nivel de riesgo del proyecto, obtenido a partir de datos de empresas cotizadas, del mismo sector/actividad y ámbito geográfico, y no diversificadas (pure players). Para ello suele utilizarse un modelo de valoración de activos financieros como es el Capital Asset Pricing Model (CAPM), que asume que los mercados financieros eficientes no retribuyen la totalidad del riesgo, sino únicamente la parte no idiosincrática o sistemática del mismo - aquella que no puede eliminarse mediante diversificación-y en proporción a su correlación con el conjunto del mercado medida a través de un parámetro beta $(\beta)$ :

$$
r_{F P}=r_{L R}+\beta \cdot P R M
$$

donde la prima de riesgo de mercado PRM es el exceso de la rentabilidad esperada del mercado en su conjunto $r_{M}$ con respecto a la tasa libre de riesgo $r_{L R}$, esto es:

$$
P R M=r_{M}-r_{L R}
$$

El método de los comparadores incluye los siguientes pasos para el cálculo de la $\beta$ (y, por tanto, $r_{F P}$ ) nocional:

- Escoger $m$ comparadores representativos y, para cada uno de ellos, calcular su beta desapalancada $\beta_{U i}$ aplicando a $\triangleright$ 
su beta apalancada $\beta_{i}$ la fórmula de Hamada, que asume que esta aumenta linealmente con la proporción entre deuda y fondos propios, para reflejar así un mayor riesgo generado por la financiación ajena, de acuerdo con la segunda proposición de Modigliani-Miller:

$$
\beta_{U_{i}}=\frac{\beta_{i}}{1+\frac{D_{i}}{F P_{i}} \cdot\left(1-T_{i}\right)}
$$

- Calcular la beta desapalancada promedio:

$$
=\frac{\sum_{i=1}^{m} \frac{\beta_{i}}{1+\frac{D_{i}}{F P_{i}} \cdot\left(1-T_{i}\right)}}{m}
$$

- Calcular el ratio de apalancamiento (gearing) regulatorio o nocional, como promedio de los $R A_{i}=D_{i} /\left(D_{i}+F P_{i}\right)$ :

$$
R A=\frac{\sum_{i=1}^{m} R A_{i}}{m}=\frac{\sum_{i=1}^{m} \frac{D_{i}}{D_{i}+F P_{i}}}{m}
$$

- Calcular la beta reapalancada promedio mediante la fórmula de Hamada:

$$
\beta=\left[1+\frac{R A}{1-R A} \cdot(1-T)\right] \cdot \beta_{U}
$$

Siendo $r_{D}$ el coste de la deuda nocional, se tiene finalmente que el WACC regulatorio es:

$$
\begin{gathered}
W A C C=R A \cdot r_{D} \cdot(1-T)+(1-R A) . \\
\cdot\left[r_{L R}+\beta \cdot P R M\right]
\end{gathered}
$$

con:

$$
\begin{aligned}
\beta= & {\left[1+\frac{R A}{1-R A} \cdot(1-T)\right] . } \\
& \sum_{i=1}^{m} \frac{\beta_{i}}{1+\frac{D_{i}}{F P_{i}} \cdot\left(1-T_{i}\right)} \\
& \frac{\frac{m}{m}}{}
\end{aligned}
$$

Este esquema general de tipo bottom-up constituye una metodología de gran aceptación para el cálculo del WACC (y, por tanto, la $T R F$ razonable) sobre la cual los matices se refieren a la elección de los comparadores, el periodo y la frecuencia de observación, la base o referencia y la forma de cálculo de cada uno de los parámetros del modelo (a saber, $r_{L R}, P R M$, $\beta_{i}, R A, r_{D}$ y $\left.T\right)$, así como el horizonte regulatorio en que, de acuerdo con la legislación sectorial específica, resulte de aplicación el valor así calculado. En el siguiente epígrafe se describen algunas de las prácticas habituales al respecto, sin entrar en los aspectos más técnicos.

\section{Aspectos metodológicos}

\subsection{Sobre la utilización del CAPM}

Por su simplicidad y objetividad, el CAPM se prefiere frente a otros modelos de valoración de activos financieros, como son el basado en el arbitraje (Arbitrage Pricing Theory -APT-) de Ross o el de los tres factores de Fama y French (3F-FF). El APT modela la prima de riesgo esperada para un activo dado como combinación lineal de diversos factores macroeconómicos, representativos de la dinámica económica general, significando sus coeficientes la sensibilidad particular de aquella frente a cada factor. Por su parte, el modelo 3F-FF corrige el CAPM para tener en cuenta el mejor comportamiento relativo que muestran las acciones de baja capitalización (vs de alta capitalización) y las acciones de valor (vs de crecimiento).

Entre los parámetros del CAPM cabe distinguir aquellos económicos generales $\left(r_{L R}, P R M\right.$ y $T)$ de aquellos específicos de empresa (RA, $\beta$ y $\left.r_{D}\right)$. Para el cálculo de estos últimos, y en función de la información disponible y de si el $\triangleright$ 
fin es calcular un WACC genérico para una actividad empresarial o uno particular para una empresa concreta, son posibles tres enfoques: de mercado -el basado en comparadores del que se viene hablando-, individual — valores del negocio o de la empresa en cuestión- o de grupo empresarial —valores del grupo al que pertenezca la empresa en cuestión-. Caben también metodologías mixtas en la que se use un criterio diferente para cada parámetro específico, utilizando para algunos de ellos valores comparables y para otros valores propios individualizados.

\subsection{Sobre la tasa libre de riesgo}

El activo libre de riesgo es aquel sin exposición a la sistemicidad del mercado, siendo su riesgo completamente diversificable. Como activo de referencia libre de riesgo suele tomarse el bono soberano (bono del Estado a diez años), ajustando al alza su rentabilidad por los programas de expansión cuantitativa (Quantitative Easing -QE-) en tanto que estos resulten en una infraestimación del riesgo-país.

Su valor $r_{L R}$ a incorporar en el WACC es un promedio de una serie histórica representativa de cotizaciones expresadas en porcentaje de TIR teórica (Yield To Maturity -YTM-):

$$
\begin{gathered}
P_{\text {bono }}=\frac{\text { CUPON }}{1+Y T M}+\frac{\text { CUPON }}{(1+Y T M)^{2}}+\ldots+ \\
+\ldots+\frac{\text { CUPON }+ \text { VALOR FACIAL }}{(1+Y T M)^{\text {ANNOS HASTA VCTO }}}
\end{gathered}
$$

\subsection{Sobre la prima de riesgo de mercado}

Un primer aspecto importante es la definición del alcance geográfico del mercado con respecto al cual se estima la prima de riesgo, lo que en última instancia marca los índices de referencia que se van a utilizar en el cálculo. Si pertenecen a diferentes países los mercados en los que cotizan los comparadores, una prima de riesgo de mercado $P R M$ única a efectos del cálculo del WACC puede obtenerse como media ponderada por las capitalizaciones bursátiles de cada una de las primas de mercado con respecto al bono soberano del correspondiente país. Otra alternativa es ponderar estos últimos por separado de las rentabilidades de mercado, utilizando como pesos el PIB de cada país, para luego calcular la diferencia entre ambas medias.

Como enfoques habituales para la estimación de la prima de riesgo de mercado esperada de cada país, los numerosos proveedores externos de esta información utilizan tanto los basados en rentabilidades ex post o históricas (métodos Ibottson y Dimson, Marsh \& Staunton -DMS-), que tabulan medias aritméticas/rentabilidad con interés simple y medias geométricas/rentabilidad con interés compuesto) como los basados en rentabilidades ex ante o previsionales, obtenidas mediante modelos de valoración de dividendos o mediante encuestas de expectativas a expertos (método de Pablo Fernández).

En una inferencia de PRM implícitas se asumen ciertas hipótesis sobre el comportamiento de las rentabilidades de mercado que simplifican su estimación a partir de cotizaciones $u$ otros indicadores bursátiles (como la relación precio/beneficio PIE O PER y los dividendos) disponibles de los índices representativos:

- Rentabilidades históricas (Implied Pricing Method del CFA Institute, para países sin datos tabulados). Si para la cartera de mercado es $P_{M}$ la cotización y $\triangleright$ 
$B P A_{M}$ el beneficio por acción, en una hipótesis de ausencia de crecimiento es:

$$
P R M=\frac{1}{P E R_{M}}-r_{L R}=\frac{B P A_{M}}{P_{M}}-r_{L R}
$$

- Previsiones futuras (Dividend Growth Model-DGM/Gordon-Shapiro; método también utilizado para la identificación a partir de YieldCos de valores suelo para $\left.r_{F p}\right)$. Si para la cartera de mercado es $D I V_{M}$ el último dividendo por acción y $g$ su tasa de crecimiento anual esperado, tendremos:

$$
P R M=\frac{D I V_{M} \cdot(1+g)}{P_{M}}+g-r_{L R}
$$

Al igual que con la tasa libre de riesgo, la representatividad del valor único de la prima de mercado estimada a partir de datos históricos exige considerar una serie temporal suficientemente extensa.

\subsection{Sobre los tipos impositivos general y de los comparadores}

Por las dificultades prácticas de estimar tipos efectivos, es recomendable el uso de tipos estatutarios -nominales- del impuesto de sociedades en cada país (25\% en España) publicados por fuentes como la OCDE.

Cabe una formulación vanilla del WACC que considere $T=0$, esto es, que pondere el coste de la deuda antes de impuestos y el de los fondos propios después de impuestos, lo que en una recuperación de costes por bloques exigiría la inclusión de uno adicional de naturaleza impositiva.

\subsection{Sobre la elección de los comparadores}

En cuanto al ámbito geográfico cercano para la elección de los comparadores, es habitual excluir aquellos países con baja calificación crediticia/rating.

En general, puede ser difícil encontrar comparadores puros no diversificados, por lo que la comparabilidad revestirá siempre cierta subjetividad. De hecho, de la imperfección de los comparadores puede resultar la necesidad de considerar en la tasa de retribución, y como diferencial por encima del WACC nominal después de impuestos, una prima por riesgos específicos adicionales $P R E A$, a estimar lo más informadamente posible para no menoscabar por opacidad la metodología:

$$
T R F=\frac{W A C C+P R E A}{1-T}
$$

La muestra de comparadores se suele depurar en cálculos particulares para la eliminación de valores atípicos —outliers - (por ejemplo, a la hora de calcular el ratio de apalancamiento regulatorio) o de acuerdo con el ratio de umbrales prefijados (por ejemplo, de liquidez a la hora de calcular la beta, o de calificación crediticia a la hora de calcular el coste de la deuda).

\subsection{Sobre el ratio de apalancamiento regulatorio}

Para los valores de $F P_{i}$ y $D_{i}$ pueden utilizarse tanto valores de mercado como contables, siendo lo habitual utilizar de mercado - capitalización bursátil- para los fondos propios y contables para la deuda, promediando datos históricos de un periodo suficientemente representativo. En el valor de la deuda cabe contemplar tanto la de largo como la de corto plazo, neta de efectivo y otros activos equivalentes - caja excedentaria-.

El cálculo del apalancamiento regulatorio o nocional $R A$ como promedio del de los $\triangleright$ 
comparadores excluyendo valores atípicos tiene sentido en tanto que estos tienen incentivos para la optimización de su estructura financiera, a la vista del trade-off que suponen los mayores escudo fiscal y riesgo de insolvencia (y, por tanto, mayor exigencia de rentabilidad a los fondos propios) asociados con mayores tasas de endeudamiento. Es decir, el apalancamiento nocional así establecido es razonablemente óptimo.

En lugar de una media aritmética de los ratios de apalancamiento de los comparadores, alternativamente cabe calcular una media ponderada para asignar así más peso a los más grandes:

$R A=\sum_{i=1}^{m}\left[\frac{D_{i}+F P_{i}}{\sum_{i=1}^{m}\left(D_{i}+F P_{i}\right)}\right] \cdot R A_{i}=\frac{\sum_{i=1}^{m} D_{i}}{\sum_{i=1}^{m} D_{i}+\sum_{i=1}^{m} F P_{i}}$

Con un enfoque individual, para la empresa concreta que se esté calculando el WACC se utilizará su propio ratio de apalancamiento.

\subsection{Sobre la beta}

La beta de cada comparador se calcula según el CAPM a partir de un ajuste de regresión lineal entre $r_{i}-r_{L R}$ y $P R M$ del que resulta $\beta_{i}=\operatorname{Cov}\left(r_{i}, r_{M}\right) / \operatorname{Var} r_{M}$. Lo que requiere disponer de información histórica sobre cotizaciones de cada comparador y del índice del mercado local en el que cotice, puesto que, por definición, las rentabilidades son variaciones porcentuales de precios dentro de un periodo concreto. Una alternativa al cálculo propio es el recurso a valores publicados por proveedores de información financiera reconocidos.

En ocasiones, las observaciones se someten al ajuste bayesiano de Blume, que busca mitigar el error en la estimación $p$ originado por una insuficiencia de datos asumiendo que las betas convergen a uno (valor de la beta del mercado en su conjunto) en una reversión a la media en el largo plazo por efecto de la diversificación:

$\beta_{i}=\beta_{i}^{\text {observada }} \cdot p+\beta_{M} \cdot(1-p) \operatorname{con} p=2 / 3$ y $\beta_{M}=1$

En el cálculo de $\beta_{U}$ como promedio de las $\beta_{U_{i}}$ desapalancadas se suelen excluir aquellos comparadores que no superen un test de liquidez basado en diferenciales medios de compraventa (bid-ask spread).

Que exista un riesgo de impago de la deuda o una correlación entre los precios de los bonos y las acciones - por verse afectados ambos por los tipos de interés- supone admitir cierto valor de la beta de la deuda, para el cual se suele tomar $\beta_{D}=\beta_{D_{i}}=0,1$. En este caso, y como alternativa a la fórmula de Hamada para desapalancar y reapalancar betas, puede utilizarse la fórmula de Miller, según la cual:

$$
\beta_{U_{i}}=\frac{D_{i}}{D_{i}+F P_{i}} \cdot \beta_{D_{i}}+\frac{F P_{i}}{D_{i}+F P_{i}} \cdot \beta_{i}
$$

y, por tanto:

$$
\beta=\frac{\beta_{U}-\frac{D}{D+F P} \cdot \beta_{D}}{\frac{F P}{D+F P}}=\frac{\frac{\sum_{i=1}^{m} \beta_{U_{i}}}{m}-R A \cdot \beta_{D}}{1-R A}
$$

\subsection{Sobre el coste de la deuda}

El coste de la deuda $r_{D}$ se expresa a menudo en función de una prima $P R D$ por encima de la tasa libre de riesgo, de forma que $r_{D}=r_{L R}+P R D$.

Como activos de referencia de deuda se suelen tomar bonos corporativos a diez años o, de no disponerse de estas emisiones, instrumentos financieros asociados al impago de la deuda, del tipo permuta de cobertura de $\triangleright$ 
incumplimiento crediticio (Credit Default Swap -CDS-) (específico de cada emisor), que se adicionan como diferencial de interés sobre el índice de permuta de tipos de interés (Interest Rate Swap-IRS-) (interés de referencia en las emisiones a tipo fijo). A partir de una serie de datos histórica suficientemente representativa se promedia su coste expresado como porcentaje de YTM calculado (en el caso de las emisiones de bonos) o de cotización (en el caso IRS + CDS).

Con un enfoque de comparadores, el coste de la deuda a utilizar en el WACC se calculará como promedio de los de cada miembro de la muestra, sin tener en cuenta aquellos de calificación crediticia dudosa - sin grado de inversión (investment grade)- Con un enfoque individual, para la empresa concreta que se esté calculando el WACC se utilizará su propio coste de la deuda.

\section{Rentabilidad efectiva}

Se ha visto cómo en el modelo de referencia para la recuperación total de costes es $T I R=T R F$. En estas condiciones, la fijación de la tasa de retribución financiera en el nivel del WACC regulatorio o nocional asegurará la financiabilidad del proyecto si y solo si ocurre que los parámetros de financiación del mismo (a saber, apalancamiento, coste de la deuda y tipo impositivo) coinciden exactamente con los del comparador nocional. De lo contrario, el coste de capital reconocido $\mathrm{VNI} \cdot W \mathrm{WCC}_{\mathrm{Al}}$ se desviará del coste de capital real $V N I \cdot W A C C_{A l}^{\text {real }}$ en sus dos componentes, tanto el explícito de deuda $V N I \cdot R A^{\text {real }} \cdot r_{D}^{\text {real }}$ como el implícito de fondos propios $V N I \cdot\left[\left(1-R A^{\text {real }}\right) /\left(1-T^{\text {real }}\right)\right] \cdot r_{F P}$.

Teniendo en cuenta esta desviación de costes de naturaleza financiera, así como las otras posibles ya mencionadas debidas a incentivos y penalizaciones regulatorias (por eficiencia en costes, por extensión de la vida útil, por mejora en la calidad del servicio o por mejora de la productividad) que rompen la igualdad $T I R=T R F$, en los modelos retributivos RoR-RAB cabría hablar de una rentabilidad efectiva. En efecto, siendo $F C^{S}$ el vector de flujos de caja libres en el caso base cost-plus, y siendo $F C^{\text {real }}$ el vector de flujos de caja libres realmente cosechados, se tendría:

- En condiciones nocionales:

$$
\begin{gathered}
\operatorname{VAN}\left(F C^{S}, T I R\right)=0 \text { con } T I R=T R F y \\
T R F=W A C C
\end{gathered}
$$

- En condiciones reales:

$$
\begin{gathered}
V A N\left(F C^{\text {real }}, W A C C^{\text {real }}\right)>0 \text { con } T I R>T R F \\
\text { y } T R F=W A C C
\end{gathered}
$$

La primera de las anteriores formulaciones, que puede reescribirse como $\operatorname{VAN}\left(F C^{S}\right.$, $W A C C)=0$ o $T I R=W A C C$, es una formalización estilizada de la idea de rentabilidad razonable. La segunda expresión refleja una situación particular de rentabilidad efectiva superior, o creación de valor, a través de la generación de flujos de caja positivos, por encima de los del caso base, y con origen último en eficiencias operativas y financieras que un buen diseño regulatorio ha de incentivar.

La rentabilidad efectiva puede expresarse, para mayor transparencia y facilidad de cálculo, en una versión adaptada del RoE como es la rentabilidad del capital regulatorio (Return on Regulatory Equity -RoRE-), o beneficio después de intereses e impuestos $B D I /$ dividido por los fondos propios regulatorios o nocionales (aquellos calculados a partir del valor $\square$ 
neto de inversión considerando el ratio de apalancamiento regulatorio):

$$
R o R E=\frac{B D I I}{(1-R A) \cdot V N I}
$$

Una buena práctica regulatoria, orientada a una mejor fijación del WACC nocional, es el análisis de rangos $R o R E$, consistente en el cálculo prospectivo de la rentabilidad financiera efectiva de cada empresa regulada, en sendos escenarios favorable y desfavorable, para así explicar y controlar, en términos de cada una de las desviaciones citadas, las causas de la divergencia entre los valores posibles del RoRE y su valor reconocido $r_{F P}$. Este análisis de riesgos, junto con un estudio más completo de ratios contables, permite caracterizar el nivel de resiliencia financiera de las empresas reguladas. En este sentido, un modelo retributivo RoR-RAB puede también contemplar penalizaciones explícitas al incumplimiento del principio de prudencia financiera por desviaciones con respecto a los valores de referencia que se establezcan para los ratios contables.

Relacionada con la rentabilidad efectiva es la nada trivial cuestión de la definición del periodo regulatorio, de cuya duración depende el efecto de aquella, en tanto que periodos regulatorios largos incentivan dinámicamente la eficiencia, mientras que los cortos favorecen el traslado de ganancias en esta a los usuarios.

\section{Conclusiones}

El establecimiento de rentabilidades en sectores regulados es un aspecto clave del que depende la efectividad de la acción regulatoria y que, en todo caso, habrá de seguir un proceso transparente y objetivo que aporte certidumbre sin dejar lugar a arbitrariedad alguna.
En el presente artículo se ha descrito someramente la metodología de estimación prospectiva del WACC nominal basada en comparadores para la fijación de tasas de retribución financiera razonables en entornos regulados. La razonabilidad en términos de financiabilidad de las rentabilidades así calculadas resulta de su naturaleza de coste de oportunidad ajustado al riesgo. Comentar que el término «financiabilidad» se ha utilizado como acepción parcial de un concepto más amplio que asocia el acceso a la financiación con la calificación crediticia, cuestión que no tiene en cuenta exclusivamente métricas financieras, sino también otros aspectos cualitativos del entorno regulatorio, las operaciones o la gestión del modelo de negocio, entre otros.

Por aplicación del WACC regulatorio antes de impuestos (o CMPCAl en su acrónimo en español) como tasa de retribución financiera nominal a valores de inversión netos de amortización, se obtiene un coste de capital que incluye fondos tanto propios como ajenos, lo que habrá de tenerse en cuenta para evitar una doble recuperación de los intereses. Este coste de capital, que en la contabilidad financiera luce solo parcialmente -al constar en esta únicamente los costes financieros ajenos explícitos-, deberá reflejarse en una contabilidad analítica cuya llevanza, en ciertos casos, es obligatoria. En situaciones de comparabilidad imperfecta, la tasa de retribución financiera puede incorporar como prima un diferencial por riesgos específicos adicionales, lo que deberá hacerse en todo caso razonadamente y con precaución.

Que el WACC nocional se manifieste sin más como TIR dependerá, por un lado, de que los métodos de amortización y valoración de activos del modelo de recuperación de costes no introduzcan distorsiones (esto es, de la $\triangleright$ 
mecánica del modelo financiero) y, por otro, de que no haya otros componentes retributivos adicionales a modo de incentivo o penalización, incluyendo entre estos uno de naturaleza financiera que capture el grado de similaridad con el estándar nocional de financiación.

Dentro de las numerosas variantes de cálculo del WACC existentes, la legitimidad de la que el regulador escoja dependerá de lo participativo de su diseño, de la publicidad de su nivel de detalle y de una accesibilidad a los parámetros últimos tal que permita su replicabilidad.

Como comentario final, precisar que, si bien la metodología presentada sacrifica ortodoxia y complejidad en aras a la practicidad, pese a sus limitaciones puede afirmarse que las rentabilidades razonables que proporciona son estimaciones razonadas (educated guesses) que, sin duda, obedecen al adagio «se non è vero, è ben trovato».

\section{Bibliografía}

Resolución de 6 de mayo de 2021, de la Comisión Nacional de los Mercados y la Competencia, por la que se publica la Resolución relativa al establecimiento de la nueva metodología de cálculo del coste del capital medio ponderado de los operadores declarados con poder significativo de mercado. Boletín Oficial del Estado, n.. 120, de 20 de mayo de 2021, pp. 61990 a 61991. https://boe.es/diario_boe/txt.php?id= BOE-A-2021-8439

Circular 2/2019, de 12 de noviembre, de la Comisión Nacional de los Mercados y la Competencia, por la que se establece la metodología de cálculo de la tasa de retribución financiera de las actividades de transporte y distribución de energía eléctrica, y regasificación, transporte y distribución de gas natural. Boletín Oficial del Estado, n. ${ }^{\circ}$ 279, de 20 de noviembre de 2019, pp. 127725 a 127734. https://www.boe.es/diario_boe/txt.php ?id=BOE-A-2019-16639 